\title{
Effects of tilt angle on droplets condensation on hydrophobic surface
}

\author{
Zhenhua He ${ }^{1}$, Shuxiang Wang ${ }^{1 *}$, Hongjie Liu ${ }^{1}$, Junjie Tong ${ }^{1}$, Zuyang $\mathrm{He}^{1}$ \\ ${ }^{1}$ Guangzhou Maritime University, Guangzhou, 510725, China
}

\begin{abstract}
Condensation surface modification was an important research direction to realize droprise condensation and erhance heat transfer. In order to explore the condensation process and mechanism of droplets on the hydrophobic surface, the condensation process of droplets on the natural Donghu lotus leaves with tilt angle of $30^{\circ}, 60^{\circ}$ and $90^{\circ}$ were studied. The results show that the tilt angle had a significant effect on the droplets condensation behavior on Donghu lotus leaves hydrophobic surface. Increasing the tilt angle would short the detachment time of condensate droplets. The growth rate of droplets increased with the increase of hydrophobic surface tilt angle. And the condensation droplets diameter also decreased with the increase of incl ination angle.
\end{abstract}

\section{Introduction}

Condensation widely exists in scientific research and industrial application, and plays an important role in energy, power, thermal management, seawater desalination and environmental control. Because the heat transfer coefficient of dropwise condensation is several times or even an order of magnitude larger than that of film condensation under the same other conditions, it is very important to maintain the dropwise condensation state in the field of condensation and heat transfer enhancement.

The study of dropwise condensation began with the first discovery of Schmidt et al in 1930s [1]. Materials with low surface energy, high thermal conductivity, long dropwise condensation life and feasible processing technology were the key to realize dropwise condensation. The current methods to achieve dropwise condensation could be summarized into three methods such as noble metal plating [2,3], condensation surface modification [4-9], and addition of organic accelerators $[10,11]$. The development of materials with better hydrophobic properties was the main research direction to achieve dropwise condensation [12]. In recent years, the method of droplet condensation based on gradient energy surface had become a research hot spot [13-14]. However, the mechanism of dropwise condensation by this method had not been thoroughly studied, and long-term continuous droplet condensation could not be achieved. So that it had not been able to achieve large-scale application in industry. The rescarch on the dropwise condensation process was still a key rescarch tield.

In this paper, a high-speced camera syslem was used to study the condensation process of natural hydrophobic surface under diffierent tilt angles. Effects of tilt angle on droplets formation, development, combination and separation during the condensation process were focus on analysis.

\section{Experimental systems and Experimental materials}

\subsection{Experimental system}

The experimental system was shown in Figure 1. The system was mainly composed of cold source, high-speed camera, LED cold light source and three-dimensional adjustment device. In order to reduce the thermal resistance between the lotus leaf and the cold source, a layer ofthermal conductive silica gel was applied between the lotus leaf and the cold source. The cold source was composed of ice water mixture to provide a temperature of $0^{\circ} \mathrm{C}$. The natural lotus leaf was in close contact with the cold source. The surface temperature of the lotus leaf was close to $0^{\circ} \mathrm{C}$. Because the surface temperature of the lotus leaf was lower than the dew point temperature of the environment, the water vapor in the air would condense on the surface of natural lotus leaf.

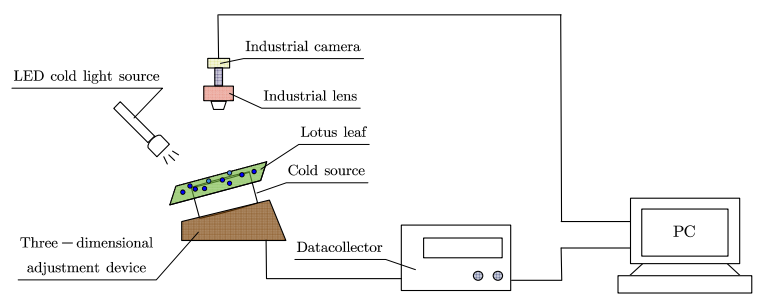

Figure 1 Schematie diagram of experimental system 


\subsection{Experimental materials and its preparations}

In order to verify its hydrophobicity, the average static contact angle and rolling angle of water droplets on horizontal lotus leaves were $148.3^{\circ}$ and $21.30^{\circ}$ respectively, as shown in Figure 2.

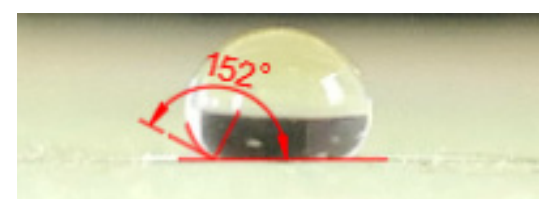

Figure 2 Static contact angle and rolling angle of drops on the lotus leaf

\section{Experimental results and analysis}

\subsection{Condensation process}

The condensation process on the lotus leaf surface at the tilt angles of $30^{\circ}, 60^{\circ}$ and $90^{\circ}$ (a, b and c, respectively) was recorded, as shown in Fig 3. From the pictures, the process of droplet generation, growth, combination and detachment could be obscrved. It could be seen that when the time $\mathrm{t}=10 \mathrm{~min}$, the droplet coverage gradually increased with the condensation process. When the droplet coverage reached a certain value on the surface, the droplet diameter began to increase, and droplet coalescence occured, which was the droplet development stage.

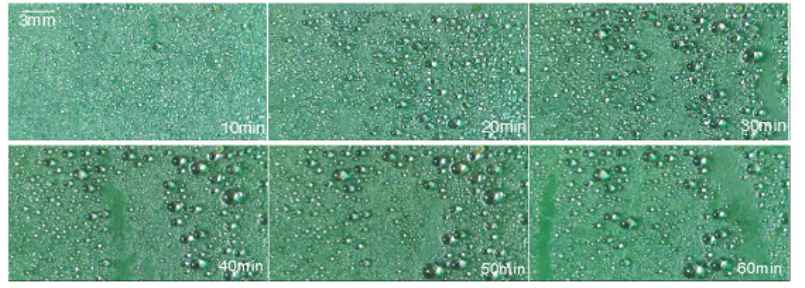

(a) $\alpha=30^{\circ}$

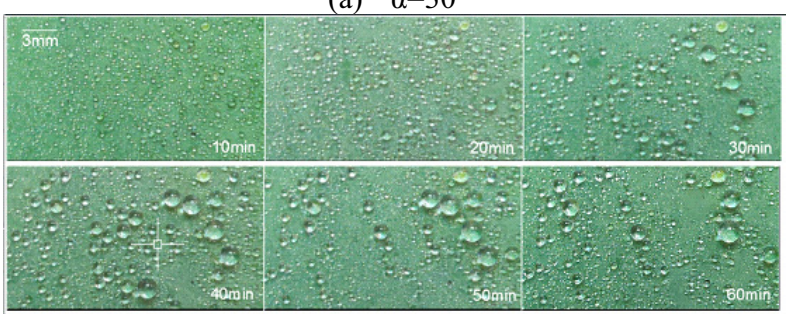

(b) $\alpha=60^{\circ}$

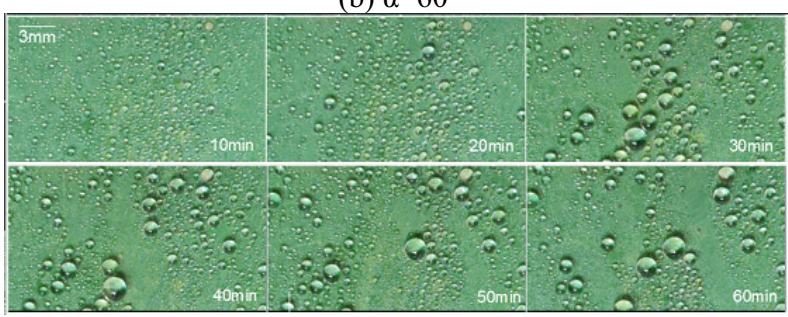

(c) $\alpha=90^{\circ}$

Figure 3 Condensation process on the surface of the lotus leaf

In Figure 3, when the droplet diameter increased to a certain value, a large mumber of droplets began to merge, and their diameters increased significantly. At this time, the droplets were in the stage of growth and merging. In this process, the droplet diameter continued to increase by combining small droplets and condensed steam. When the droplet started to fall off from the surface and refresh the lotus leaf surface, the condensate droplet began to take off. Figure 3 showed that small droplets formed by newly condensation on the path of droplet take off. In this condensation process, the droplets had undergone a process of generation, growth, merging, and take off from the surface.

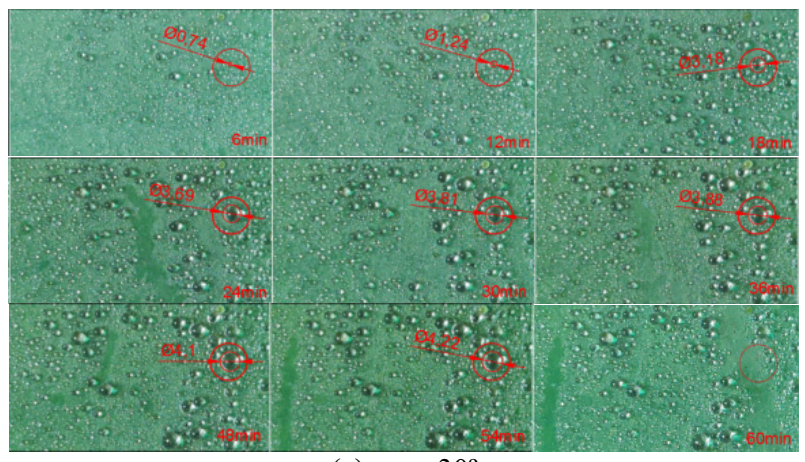

(a) $\alpha=30^{\circ}$

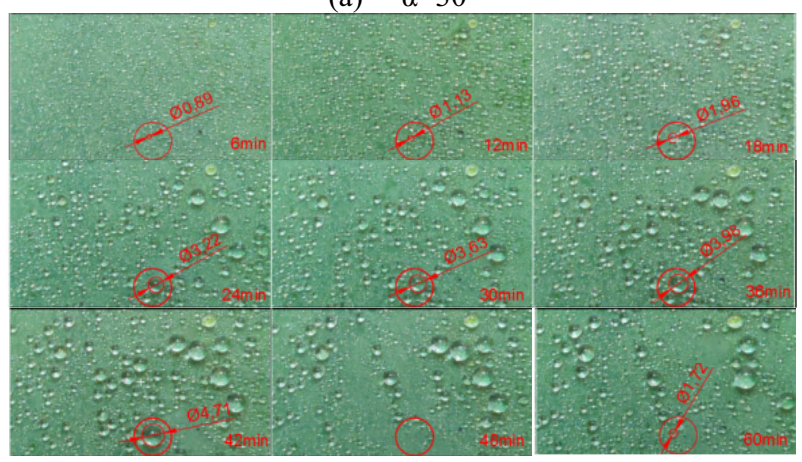

(b) $\alpha=60^{\circ}$

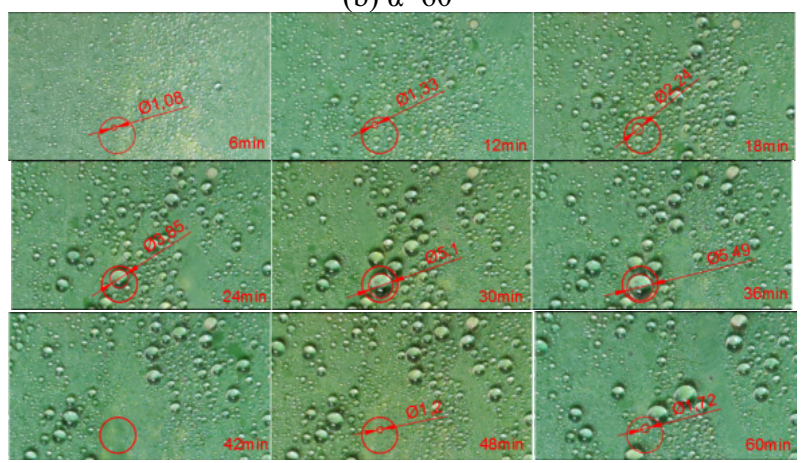

(c) $\alpha=90^{\circ}$

Figure 4 Variation of droplet diameter with time at the same slope angle

\subsection{Analysis of the condensation process}

From the process of droplet condensation, it could be seen that the period time relationship of droplet condensation under different tilt angles was as follows:

$$
t_{\mathrm{a}}>t_{\mathrm{b}}>t_{\mathrm{c}}
$$

Taken any point on the surface of the lotus leaf. Observed and recorded the change in the diameter of the same droplet within 1 hour at an interval of $6 \mathrm{~min}$ from the starting time. As shown in Figure 4, the critical 
detachment diameter and hysteresis contact angle of the droplet were measured and analyzed.

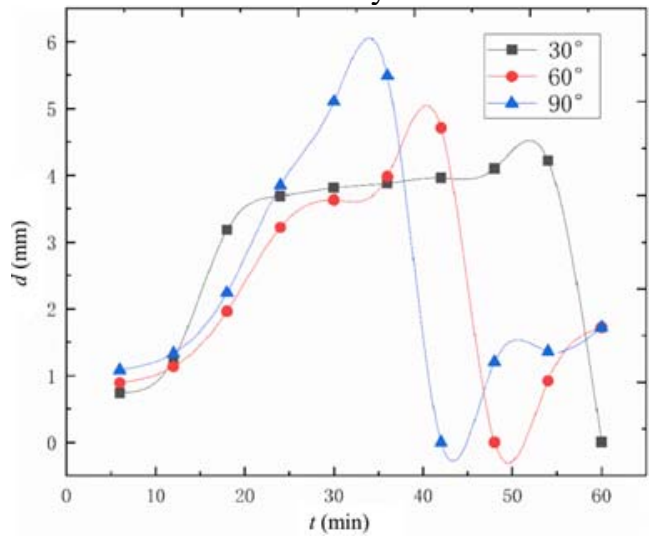

Figure 5 Change of droplet diameter with time

\subsection{Droplet departure diameter analysis}

Figure 5 showed the change of the diameter of the droplet with time when the surface was inclined at different surface inclination angles. It can be seen from Figure 5 as follows:

(1) At the initial stage of condensation, the growth rate of droplet diameter under three different tilt angles was nearly the same. The difference occured during 12 min and $18 \mathrm{~min}$, the growth rate of droplet suddenly increased at the tilt angle $\alpha=30^{\circ}$. During this time, the droplet $\left(\alpha=30^{\circ}\right)$ was in the growth merging stage. Then its rising trend of curve was close to the level. The droplet growth rate of tilt angle $\alpha=60^{\circ}$ and $\alpha=90^{\circ}$ were roughly the same. But the rising speed of curve with $\alpha=60^{\circ}$ was slightly faster than the curve with $\alpha=90^{\circ}$. From Figure 5, the droplet growth rate increased with the tilt angle increasing.

(2) At the different tilt angles of $30^{\circ}, 60^{\circ}$ and $90^{\circ}$, the time when the droplet condensation curves reached the highest point was $54 \mathrm{~min}, 42 \mathrm{mir}$ and $36 \mathrm{~min}$, respectively. Table I showed the critical detachment diameter and lag contact angle of droplets with different tilt angles.

Table 1 Critical detachment diameter and hysteresis contact angle at different tilt angles

\begin{tabular}{ccc}
\hline $\begin{array}{c}\text { Surface tilt } \\
\text { angle }\left({ }^{\circ}\right)\end{array}$ & $\begin{array}{c}\text { Critical detachment } \\
\text { diameter }(\mathrm{mm})\end{array}$ & $\begin{array}{c}\text { Hysteresis contact } \\
\text { angle }\left({ }^{\circ}\right)\end{array}$ \\
\hline 30 & 2.69 & 68.7 \\
60 & 2.56 & 38.3 \\
90 & 2.33 & 22.3 \\
\hline
\end{tabular}

(3) The relationship between the critical detachment diameters $(R)$ of droplets on three groups of surfaces with different tilt angles was $R_{30^{\circ}}>R_{60^{\circ}}>R_{90^{\circ}}$.

(4) The relationship between hysteresis contact angle $\left(\theta_{\mathrm{h}}\right)$ was $\theta_{\mathrm{h}, 30^{\circ}}<\theta_{\mathrm{h}, 60^{\circ}}<\theta_{\mathrm{h}, 90^{\circ}}$

By the analysis of Figure 5, it could be got that the hysteresis contact angle of the condesation droplet decreased with the increase of the tilt angle hydrophobic surface. The droplets detachment was easier at lagerer tilt angle hydrophobic surface, which led to the smaller critical drop liameter. The growth rate of droplet diameter was faster at lager tilt angle, so the time for droplet diameter to reach the critical drop diameter was shoten.

\section{Conclusions}

Experimental research on the droplet condensation process on hydrophobic surface with natural lotus leaves was carried out. Hydrophobic surface tilt angle effects on growth rate, critical departure diameter and contact angle hysteresis of droplets condensation were analyzed in detail. The following conclusions were obtained:

(1) The growth rate of the condensation droplet diameter increased with the hydrophobic surface tilt angle increasing.

(2) During the condensation process, the condensation droplet detachment appeared firstly on the hydrophobic surface with the tilt angle of $90^{\circ}$.

(3) When the tilt angle increased, the droplet detachment diameter decreased and the droplet contact angle hysteresis also decreased.

\section{Acknowledgments}

The authors thank for the financial support by the National Natural Science Foundation of China with the contract numbers of 51806041, and the Guangdong Science and Technology Project (2018A0303130013).

\section{References}

1. Gao Xing-Cun. Preparation of carbon nanotube arrays on stainless steel surface and study on heat transfer performance of droplet condensation [D]. Northeast Petroleum University,2018.

2. Wang Xian-Lin. Experimental study on droplet condensation [D]. Central South University,2005.

3. Hu Guo-Qiang, Song Yong-Ji, Xu Dun-Xin \& Lin Ji-Fang. Condensation heat transfer of steam on chromium surface $[\mathrm{J}]$. Chinese Journal of Chemical Industry, 1997, 05:616-621.

4. Zhao Qi \& Lin Ji-Fang . Research progress on industrial application of drop condensation [J]. Chemical industry progress, 1991,42(2):17220.

5. Ma Xue-Hu, Xu Dun-xin \& Lin Jin-Fang. Study on surface and drop condensation of ultrathin polymer $[\mathrm{J}]$. Chinese journal of chemical industry, 1993, 44(2):165-170.

6. Ma Xue-Hu, Xu Dun-xin \& Lin Jin-Fang. Heat transfer of condensation on the surface of ultra-thin polymers for drop condensation [J]. Chinese journal of chemical industry, 1993,44(3):277-281.

7. Hu You-Sen, Huang Wei-Tang \& Sui Hai-Ming. Experimental study on drip condensation heat transfer of PTFE coating outside tank tube [J].2007.34(5):42-45.

8. Wang Xian-Lin. Experimental study on droplet condensation [D]. Central South University,2005. 
9. Huang Yan-Feng, Lv Zao-Sheng \& Bai Hong-Qiang, et al. Preparation of self-cleaning superhydrophobic materials based on styrene and methyl methacrylate copolymer $[\mathrm{J}]$. Chemical new materials, 2017,45(05):256-258.

10. Cao Shu-Long. Research on the construction of superhydrophobic nano membrane on copper surface and its Resistance to condensation and droplet condensation [D]. Suzhou University,2013.

11. Zhao Qi, Zhang Dong-Chang \& Lin Ji-Fang. A new way to realize drop condensation $[\mathrm{J}]$. Nature Journal,1991(03):232-233.

12. Tang Yuan, Song Jia \& Bai Yang, et al. Research progress on the realization of droplet condensation $[\mathrm{J}]$. Journal of Zhejiang University (Engineering Science), 2016, 52 (02):273-287+332.

13. Deng Z, Zhang C, Shen C, et al. Self-propelled Drop-wise Condensation on a Gradient Surface [J]. International Journal of Heat and Mass Transfer, 2017, 114: 419- 429

14. Chen X, Yao S, Wang Z. Evaporation of Condensate Droplets on Structured Surfaces with Gradient Roughness[J]. Journal of Heat Transfer, 2015, 137(8): $388-392$ 